\title{
The New Edge in Knowledge: How Knowledge Management Is Changing the Way We Do Business
}

\author{
Book Review
}

\author{
Author: Carla O’Dell and Cindy Hubert \\ Reviewed by: Shiva Kumar Srinivasan \\ IIPM, Chennai, India \\ shiva.srinivasan@iipm.edu
}

The main theoretical wager in this book is that knowledge management is not what it used to be. It is time to reinvent the basic principles of knowledge management and deploy them to harness knowledge more effectively in organizations. Dell and Hubert attempt to provide the basic set of ideas to make this possible. It would not be fair to merely describe this as an' attempt' given that this book is endorsed by none less than Larry Prusak, the founder of the Institute for Knowledge Management. Prusak formalizes the new principles with which this book works at the very outset when he points out that knowledge management must henceforth be understood in the language of 'flow', as 'primarily a human activity', and, as 'profoundly social'. What these three principles have in common is the fact that knowledge is not being collected as an end in itself but with the needs of the business in mind. This is because much of the knowledge that is collected remains 'unused' or becomes 'unusable' due to errors in how it is classified, stored, and retrieved. Furthermore, it is important to ensure that the cost factors in knowledge management are clearly understood. It is not cost effective to avoid knowledge management, but at the same time, inadequate protocols of knowledge management will cost more than not having a knowledge management system at all. The idea here is not be merely dazzled by the technological availability of a system, but think of 'business outcomes' rather than the structure of knowledge management per se. It is only insofar as the outcomes enable more effective business results that it will become possible to justify the cost-factors involved in setting up, maintaining, and deploying a knowledge management architecture in the firm or in an organization. While this may seem but a matter of common sense, it is often the case that the strategic rationale is forgotten when a knowledge management system is set up. And, again, it is important to ensure that the system has an effective alignment with the strategic goals of the business enterprise. Most of these systems start off well, but fall into disuse since the 'retrieval mechanisms' are not thought through properly. In the absence of effective retrieval systems, the relevant piece of knowledge - even when available - will remain unutilized or underutilized.

Prusak's principles are important because they will substantially increase the probability of actually deploying the knowledge by recognizing the human element in knowledge management. For Prusak, the knowledge exists for the end-user and not as an end in itself. Any psychological insights that we might get into the mind-set of the end-user will therefore make a huge difference to the protocols of knowledge management. So whether we are interested in formalizing a 'personal knowledge system' that knowledge workers carry in their nervous system or in a firm-wide/organization-wide knowledge management architecture, what is at stake is the point, place, and forms of deployment to facilitate value addition vis-àvis internal and external customers. A good example of end-user psychology is the notion of a 'teachable moment'. It is the point at which an employee is 'most receptive' to the idea of learning something. Dell and Hubert argue that this is the pivotal point in knowledge management. It is around this possibility of harnessing a teachable moment that the entire architecture of knowledge management should be built. This is quite different from the previous approach which was based on the idea that if you build it, they will come'. What the authors discovered as experts in this area is that while employees will come, they may not come in large enough numbers, and when they do come, they will not know how to retrieve the knowledge effectively enough to either reduce costs or to add value. It is therefore important to start with the end-user in mind rather than with the knowledge management system. Once this 'emerging principle' is understood, it leads sooner rather than later into the questions of social psychology (that constitute the community of knowledge workers) In fact, one of the best known stories about the protocols of knowledge management relates to how mechanics who repair machines prefer to learn from each other by comparing notes, swapping experiences about recalcitrant machines, and by narrativizing them in the form of stories. This is quite different from the way that they are taught to repair machines in training programs. These are the sort of insights that organizational anthropologists and social psychologists have 
been able to generate about how 'communities of practice' determine the success or failure of technology deployment in organizations. This is needless to say the case in knowledge management as well.

Retrieval mechanisms must be designed keeping these sorts of idiosyncrasies in mind if a firm is keen to ensure that the 'lessons learned' in any given realm are not lost since the cost of re-learning the same lessons repeatedly by different sets of workers can be prohibitive for a firm. The main takeaway here is that knowledge workers are not outside the process of knowledge management, but inside. An interesting analogue here is the process whereby in Total Quality Management (TQM), the inspection process is built 'into' the manufacturing process itself and is not defined as a post-production mechanism. Not only does this empower the worker, it harnesses his brain power as well, and gives him a greater sense of ownership over the product, and increases his willingness to take responsibility for matters pertaining to quality. If this principle works in TQM, it is all the more likely to work in the context of knowledge management. That is why the authors have taken the trouble to construct an effective 'business case for knowledge management'. If knowledge workers do not understand what the business is all about, they will not be able to manoeuvre within the system effectively. The relationship between the knowledge management strategy and the over-all business strategy should therefore be spelt-out to the workers. This is getting to be a precondition for gaining the buy-in of knowledge workers who don't like following instructions without understanding the strategic rationale for any course of action that is being pushed by the firm. The firm should also differentiate between 'proven' and 'emerging' knowledge management practices, and ensure that it has spent sufficient effort in actually talking to knowledge workers. This will ensure that knowledge management does not proceed along the path of standardization, but is customized in ways that make sense to the actual users of the system. Only then will it become possible to create a 'culture of knowledge sharing' rather than 'knowledge hoarding'. At this point, knowledge management becomes what is usually described as a 'techno-structural', and/or 'human process' intervention in the language of organizational development. The link to organizational development is important to note here because the tools required to develop an organization are available within the organization itself, but its significance might not be obvious to everybody. This could well be the case if the teams responsible for knowledge management and organizational development are not conferring with each other. In such a situation, the gains from knowledge management will be sub-optimal.

Knowledge management then to conclude is not an end in itself since it costs a lot to capture, codify, and construct the relevant systems. Knowledge sharing is much more important than knowledge management. In fact, knowledge management systems were invented precisely because there is inadequate sharing of knowledge in organizations either because the incentives in place do not facilitate that process or because in situations where there is a considerable turnover of employees, there is a huge loss of knowledge for the firm. All this is easier said than done, which is precisely why readers must take the trouble to read the four cases included in the last section of the book. These cases take an in-depth look at the knowledge management strategies developed by leading firms like ConocoPhillips, Fluor, IBM, and MITRE. For those who like the theoretical aspects of knowledge management it is best to read the book sequentially before arriving at the cases as illustrations of knowledge management. For those who are actually doing knowledge management in firms (directly or indirectly), it might be easier to start by sampling the cases belonging to the sectors that are closest to their situation before thinking-through the constitutive principles of knowledge management that are set out in the earlier chapters. Not only does this book succeed in showing readers the way forward in terms of the emerging practices in knowledge management, it is designed to be a resource book. Dell and Hubert are content to sign-post the resources available rather than impose themselves on the reader; hence the importance of the net-based resources of the American Productivity and Quality Centre that are constantly referred to here. This book is highly recommended for both doing courses in this area, and for anybody who wants to understand how knowledge management is becoming an increasingly important approach to organizational developmentwhether it takes the form of 'human process interventions' or 'techno-structural interventions'. The reason for this must be obvious to the reader by now: knowledge is the currency of organizational development. It is increasingly, I am tempted to add, like the definition of money, in economics: 'a medium of exchange, a store of value, and a unit of measure'. 